[20] Tapernoux. Le Lait, 928, p. 698, 795, 916.

[21] Holm-Webв. American Journal Dis. Childr., 1930.

[22] Marfan. Le Nourrisson, 1937.

[23] Maillet. Le Nourrisson, 1938.

[24] Pи́ru-Woringer. Revue Fr. Péd., 1938.

[25] Bibliogr., voir op. cit., $\mathrm{n}^{0} 1$.

[26] WRIGHT Journal of Dairy Research, 1933, p. 122, 365, 257.

[27] How Aт-WRIGHт. Le Lait, 1935, p. 438.

[28] Debré-Semelaigne. Bull. Société Péd., 1926.

[29] Bibliogr., voir P且U, Volume Jubilaire Ch. Porcher, 1932, p. 50 ; Ch. Goldberg. La fièvre du lait sec, Thèse, Paris, 1931-1932.

[30] Debré-Lelong. La Presse Médicale, 1931, 49, p. 913-918; Bulletin Société Biologique, 1931.

[31] Congrès Péd. Strasbourg. La Presse Médicale, 1931, nº 86, p. 1577.

[32] Dorlencourt. Bulletin Société Péd., 1926.

\title{
SUR LA VALEUR \\ DE LA MÉTHODE SCHMID-BONDZYNSKI-RATZLAFF
}

\author{
par \\ Dr A. J. SWAVING
}

Dans le No 181 du "Lait " (tome XIX, janvier 1939) a été publiée une note de M. le $\mathrm{D}^{\mathrm{r}}$ Florentin, intitulée : "Le dosage de la matière grasse dans les fromages " .

L'auteur prétend "que la méthode Schmid-Bondzynski-Ratzlaff fournit des résultats inférieurs à ceux de la méthode par extraction directe, qui permet d'obtenir toute la matière grasse dans son état primitif. ")

Puis il émet le vœu : "qu'on revienne sur les conclusions de la Convention de Rome du 24 avril 1934 et qu'on utilise à l'avenir, comme méthode officielle pour le dosage de la matière grasse dans les fromages, la méthode dite "au sable »."

M. Florentin demande beaucoup; cependant, je prends la liberté de faire quelques observations à sa démonstration, afin de mettre la question au point.

Comme la méthode conventionnelle doit être appliquée en cas de contestations qui pourraient surgir dans le commerce international, il faut bien qu'elle soit une méthode absolument exacte.

A cette fin, a été proposée et adoptée comme telle au Congrès international laitier, le 8 octobre 1923 à Syracuse (Etats-Unis), la méthode Schmid-Bondzynski-Ratzlaff. Elle fut, consécutivement, adoptée sans aucune opposition :

Le 10 mai 1926 à Paris (7e Congrès international de Laiterie), où je l'ai proposée au nom de la Commission internationale pour les 
fromages ; le 16 mai 1929 à Rome (Session préparatoire des méthodes chimiques); le 9 juin 1930 à Rome (Conférence diplomatique internationale pour la protection des dénominations des fromages et l'unification des méthodes d'analyse) (étaient présents pour la France : M. Lesage, directeur de l'Agriculture, M. le Dr Toubead, inspecteur général, chef du Service de la Répression des Fraudes et M. L. Dop); le 24 avril 1934 à Rome (Conférence diplomatique internationale pour l'unification des méthodes de prélèvement des échantillons et d'analyse des fromages) (étaient présents pour la France: M. Dop et M. VIToux, directeur-adjoint du laboratoire central du Ministère de l'Agriculture).

La Convention ( $\mathrm{y}$ compris la méthode Schmid-BondzynskiRatzlaff) a été ratifiée depuis par les gouvernements des Etats suivants : Allemagne, Belgique, France, Finlande, Hollande, Italie, Norvège, Suisse, Suède et Tunisie.

Or, M. Florentin est d'avis qu'il faudra revenir à la méthode (surannée) dite " au sable ".

Il pense que « le traitement brut par un acide minéral démolissait plus ou moins les glycérides constituant le beurre, et qu'ainsi une fraction de la matière grasse, transformée en glycérides et en acides solubles dans l'eau, disparaissait. ")

Pour simplifier la question dit-il : "Nous avons traité directement 10 grammes de beurre pur (matière grasse? $\mathrm{Sw}$.) par $50 \mathrm{~cm}^{3}$ d'acide chlorhydrique, densité 1,125 au voisinage de l'ébullition, pendant 1 heure. "

Mais, dans ce cas, la situation s'est modifiée : il manque la présence de la caséine qui, entrant en solution, enlève à l'acide une grande partie de son activité.

M. Florentin calcule une perte de poids du beurre d'environ $5 \%$.

Tout en remarquant que c'est une perte énigmatique, je rappelle que la méthode Schmid-Bondzynski-Ratzlaff prescrit : "trois grammes de fromage sont pesés dans un petit ballon d'environ $50 \mathrm{~cm}^{3}$; on ajoute $10 \mathrm{~cm}^{3}$ d'acide chlorhydrique de densité 1,125 ; on chauffe doucement en secouant avec précaution jusqu'à complète dissolution du fromage, pendant environ 10 minutes. " "

Enfin, M. Florentin a analysé "avec un grand soin, un même échantillon " (je souligne) de fromage (Port-Salut) par la méthode au sable, par la méthode Lezé et par la méthode conventionnelle.

Il en conclut "que les dernières méthodes ont fourni des résultats inférieurs à ceux de la méthode par extraction directe,"

D'après mon opinion, c'est un jugement très hasardeux.

Mes doutes sur la réalité des suggestions de M. Florentin m'ont décidé - n'ayant pas un laboratoire à ma propre disposi- 
tion - à prier le directeur de la Station de contrôle de la Hollande méridionale pour le beurre et le fromage, M. le $\mathrm{D}^{\mathrm{r}} \mathrm{VAN}$ DE BUNT, à La Haye, de bien vouloir soumettre la note de M. Florentin à un examen approfondi.

M. VAN DE BUNT s'en est occupé sans retard; voici le compte rendu de son étude :

"Au laboratoire de la Station de contrôle de la Hollande méridionale pour les beurres et les fromages, à La Haye, furent conduites quelques recherches qui ont prouvé qu'il ne faut pas avoir de crainte en ce qui concerne une perte en matière grasse dans l'application de la méthode Schmid-Bondzynski-Ratzlaff pour doser la matière grasse dans le fromage.

“A. - 900 milligrammes de matière grasse additionnés de $10 \mathrm{~cm}^{3}$ d'acide chlorhydrique (D. 1,19 ou $38 \%$ ) avee $1 \mathrm{~cm}^{3}$ d'eau (correction de l'acide à cause de la teneur en eau dans le fromage) furent chauffés pendant 10 minutes au bain-marie bouillant en secouant.

"Il en est résulté que 4 essais du même échantillon rendaient en moyenne $100,08 \%$ de la quantité pesée.

"La preuve blanche (sans chauffage) rendait 100,1\%.

«B. - 900 milligrammes de matière grasse de beurre furent chauffés au bain-marie bouillant avec $10 \mathrm{~cm}^{3} \mathrm{HCl}$ (D. 1,19) et $1 \mathrm{~cm}^{3}$ d'eau, pendant 10 minutes, en secouant doucement; après refroidissement, la matière grasse fut filtrée, lavée à l'eau chaude puis séchée et extraite avec de l'éther de pétrole.

" Il n'y avait pas de perte ; il fut retrouvé en moyenne (3 essais) $100 \%$ de la quantité de matière employée.

"C. - Enfin 2 grammes de matière grasse de beurre furent bouillis doucement au bain-marie avec $10 \mathrm{~cm}^{3}$ d'acide chlorhydrique (D. 1,125) pendant 1 heure en $y$ ajoutant parfois pour compenser l'évaporation de l'acide, de très petites quantités d'acide chlorhydrique.

"Après refroidissement, etc., il fut retrouvé en moyenne (2 essais) $100,25 \%$ du poids ayant servi à l'essai. »

$$
*^{*} *
$$

Cette étude justifie encore une fois la grande confiance accordée par tant de chimistes-experts à la méthode Schmid-BondzynskiRatzlaff. 